\title{
Probiotika - Zweifel an der allergieprotektiven Wirksamkeit
}

\section{Ob die frühzeitige Gabe von probiotischen Substanzen Neurodermitis oder Allergien verhindern kann, wird kontrovers diskutiert. Eine kontrollierte Studie unter Beteiligung von Säuglingen mit hohem Allergierisiko sollte jetzt Licht ins Dunkel bringen.}

\begin{abstract}
A ustralische Kinderärzte wählten 231 Neugeborene aus, bei deren Müttern allergisches Asthma bronchiale, allergische Rhinitis oder atopisches Ekzem diagnostiziert worden war und die im Pricktest auf mindestens eines von sieben häufigen Aeroallergenen reagiert hatten. Kinder von Raucherinnen oder von Müttern mit Komplikationen in der Schwangerschaft wurden ausgeschlossen. Nach der Geburt erhielten die Kinder randomisiert als Nahrungsergänzung entweder Lactobacillus acidophilus oder Plazebo für die Dauer von sechs Monaten.
\end{abstract}

Insgesamt 178 Kinder nahmen über die gesamte Beobachtungszeit an der Untersuchung teil. Nach sechs Monaten zeigten sich keinerlei signifikante Unterschiede zwischen den beiden Gruppen, was das Auftreten von atopischem Ekzem, Giemen, Husten oder von alterstypischen Infektionen betraf. Auch nach einem Jahr waren in den beiden Gruppen die Zahlen für atopisches Ekzem ohne klaren Unterschied. Ebenso war der Anteil von Kindern mit Nahrungsmittelallergien nicht signifikant verschieden. Auffällig war dagegen, dass in der Gruppe mit Probiotikaeinnahme deut- lich mehr Kinder positiv im Prickttest gegen häufige Allergene reagierten (40\% vs. $24 \%, p=0,03$ ). Ebenfalls signifikant häufiger war der kombinierte Parameter „atopisches Ekzem plus positiver Pricktest" in der Probiotikagruppe (26\% vs. $14 \%, \mathrm{p}=0,045)$.

Fazit: Kinder, die im ersten Lebenshalbjahr Lactobacillus acidophilus erhalten hatten, entwickelten in dieser Untersuchung genau so häufig ein atopisches Ekzem wie die Kinder der Kontrollgruppe. Die Zahl der durch Pricktest nachgewiesenen Sensibilisierungen lag in der Gruppe mit Probiotikaeinnahme sogar signifikant höher.

af

Taylor AL et al. Probiotic supplementation for the first 6 months of life fails to reduce the risk of atopic dermatitis and increases the risk of allergen sensitization in high-risk children: A randomized controlled trial. J Allergy Clin Immunol 2007; 119: 184-91

\section{Fisch für Mutter und Kind}

In den skandinavischen Ländern wird wesentlich mehr Fisch konsumiert als in Deutschland. Eine Studie untersuchte, ob ein früher Beginn des Fischkonsums im ersten Lebensjahr vor späteren Allergien schützen könnte.

n einer prospektiven schwedischen Kohortenstudie wurden die Daten von 4.089 Neugeborenen bis zu einem Alter von vier Jahren dokumentiert. Die Eltern füllten dazu Fragebögen zu Fischverzehr und zur Gesundheit ihrer Kinder aus, wenn die Kinder ein Alter von zwei Monaten, einem Jahr, zwei Jahren und vier Jahren erreicht hatten. Nach vier Jahren wurde außerdem eine klinische Untersuchung mit Bestimmung des spezifischen IgE gegen häufige Allergene durchgeführt.

Litten die Eltern selbst unter allergischen Erkrankungen oder entwickelten die Kinder früh ein Ekzem oder Wheezing, führte dies zu einer verspäteten Beifütterung von Fisch. Daher wurden solche Kinder aus der Endanalyse ausgeschlossen. Bei den übrigen Kindern zeigte sich, dass ein regelmäßiger Fischkonsum bereits im ersten Lebensjahr das Risiko einer Sensibilisierung oder einer

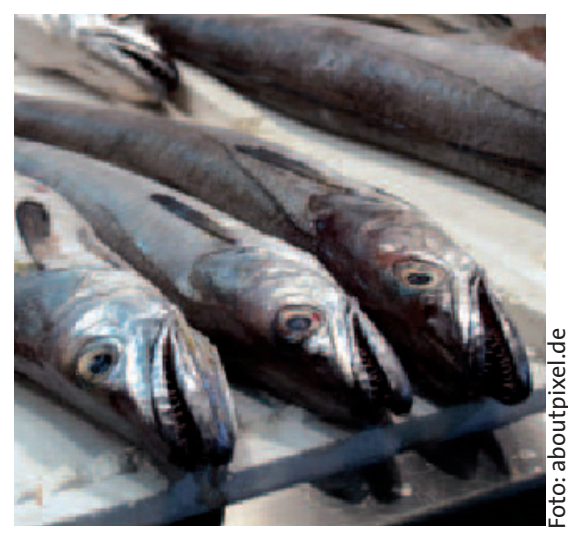

Fisch kann wohl auf die Liste der möglicherweise allergieprotektiven Nahrungsmittel gesetzt werden. allergischen Erkrankung im Alter von vier Jahren gegenüber Kindern, die nicht regelmäßig Fisch gegessen hatten, reduziert hatte (adjustierte Odds Ratio [OR] jeweils 0,76). Der protektive Effekt von Fischkost im ersten Lebensjahr war besonders deutlich bezüglich mehrfacher allergischer Erkrankungen (OR 0,56). Umgekehrt fand sich nur bei 18 der 2.614 letztlich einbezogenen Kinder spezifisches IgE gegen Fisch.

$\mathrm{Da}$ in der Auswertung andere Faktoren wie zum Beispiel die Ernährung der Mutter in Schwangerschaft und Stillzeit - Fette werden teilweise länger im Körper gespeichert - und die Art der Fischzufuhr nicht erfasst wurden, lässt die Studie leider noch viele Fragen offen.

Fazit: Erst Stillen, dann regelmäßig Fisch - das scheint eine Erfolg versprechende allergieprotektive Ernährungsstratgie für das erste Lebensjahr zu sein.

Kull I et al. Fish consumption during the first year of life and development of allergic diseases during childhood. Allergy 2006; 61: 1009-15 